\title{
Dabigatran Etexilate: What Do Hospitalists Need to Know?
}

\author{
Amer Zeidan, $\mathrm{MD}^{1}$, Bishoy Faltas, $\mathrm{MD}^{2}$, Michael Streiff, MD ${ }^{1 *}$
}

${ }^{1}$ Division of Hematology, Department of Medicine, Johns Hopkins University School of Medicine, Baltimore, Maryland; ${ }^{2}$ Division of Hospital Medicine, Department of Medicine, Rochester General Hospital, Rochester, New York.

Dabigatran etexilate (dabigatran) is a novel, oral, reversible, direct thrombin inhibitor that exhibits several advantages over warfarin for therapeutic anticoagulation. The predictable pharmacokinetic profile and minimal food and drug interactions of dabigatran allow for a fixed-dosing regimen and obviate the need for routine laboratory monitoring. Dabigatran has been approved in the United States for prevention of stroke in patients with nonvalvular atrial fibrillation and in the European Union and other countries for primary prevention of thromboembolic events after total knee or hip replacement. More indications for the use of dabigatran are under review by regulatory authorities and are undergoing active clinical investigation. Due to its rapid onset of action, dabigatran may omit the need for a parenteral anticoagulant for acute treatment of thromboembolic conditions. Because wide-scale use of dabigatran is expected in the near future, hospitalists need to familiarize themselves with this agent. The lack of a standardized reliable laboratory method to monitor the anticoagulant effects of dabigatran complicates verifying compliance, measuring the effects of drug interactions, evaluating cases of dabigatran toxicity, and conducting preoperative evaluations. The lack of an antidote to dabigatran complicates the management of toxicity and makes it largely supportive. The elimination of dabigatran is dependent on renal function, with the potential for drug accumulation and toxicity with renal impairment. The noninferiority design of the clinical trials that evaluated dabigatran and the absence of long-term safety and efficacy data and issues related to the cost effectiveness of dabigatran should all be considered when prescribing this agent. Journal of Hospital Medicine 2012;7:262-269. (c) 2011 Society of Hospital Medicine.
Vitamin $\mathrm{K}$ antagonists (VKAs) such as warfarin have been the backbone of oral anticoagulation in clinical practice since the middle of the last century. Despite their efficacy, VKAs have well-recognized limitations that have led to their underutilization in patients who would otherwise be candidates for oral anticoagulation. ${ }^{1-4}$ These limitations include a narrow therapeutic window and significant intra- and interindividual variability in dose requirements as well as numerous drug-drug and drug-food interactions. ${ }^{5-9}$ Therefore, VKAs require close laboratory monitoring to prevent excessive or under-anticoagulation, and maintaining therapeutic anticoagulation with VKAs remains a challenging task in many patients. ${ }^{2}$ It has been shown that $30 \%-50 \%$ of international normalized ratio (INR) results fall outside of the targeted therapeutic range. ${ }^{10,11}$ Consequently, it is not surprising that warfarin is a common cause of medication-related emergency room visits. ${ }^{12}$ Despite many fruitless years of searching for better alternatives, VKAs have remained the mainstay of oral anticoagulation for more than 60 years. $^{8}$

*Address for correspondence and reprint requests: Michael Streiff, MD, 1830 East Monument Street Suite 7300, Baltimore, MD 21205; Tel.: 410-614-0727; Fax: 410-614-8601; E-mail: mstrei@@jhmi.edu

Additional Supporting Information may be found in the online version of this article.

Received: June 15, 2011; Revised: September 19, 2011; Accepted: October 29, 2011

2011 Society of Hospital Medicine DOI 10.1002/jhm.996

Published online in Wiley Online Library (Wileyonlinelibrary.com).
An ideal anticoagulant would be orally administered, effective, safe, exhibit a predictable pharmacokinetic profile and a low potential for drug or dietary interactions, and therefore would not require routine laboratory monitoring. ${ }^{2,5,13}$ Other desirable characteristics would include a rapid onset of action to decrease or eliminate the need for bridging therapy, and rapid reversibility with or without an antidote. ${ }^{8,13}$ To date, no oral anticoagulant has been developed that possesses all of these desired characteristics. Dabigatran etexilate (Pradaxa, Boehringer Ingelheim Pharmaceuticals, Inc.) has recently become the first oral anticoagulant to be available for wide clinical use since the $1950 \mathrm{~s} .{ }^{14}$ In the following sections, we provide an overview of dabigatran etexilate, with a special focus on issues that are pertinent to hospitalists and the hospitalized patient.

\section{PHARMACOLOGY OF DABIGATRAN ETEXILATE}

\section{Pharmacokinetics and Pharmacodynamics of Dabigatran Etexilate}

A comparison of the pharmacokinetic (PK) and pharmacodynamic (PD) properties of dabigatran etexilate (dabigatran) and warfarin are presented in Table 1. Dabigatran etexilate (referred to from this point as dabigatran) is a prodrug of dabigatran, which blocks the terminal coagulation cascade by binding to the active site of thrombin and selectively inhibiting this critical serine protease in a dose-dependent and reversible fashion. ${ }^{15}$ Thrombin plays a central role in blood 
TABLE 1. Comparison of Warfarin and Dabigatran

\begin{tabular}{|c|c|c|}
\hline & Warfarin & Dabigatran \\
\hline Mechanism of action & $\begin{array}{l}\text { Reduces functional levels of vitamin K-dependent factors II, VII, IX, } \\
\text { and X by inhibiting vitamin K epoxide reductase }\end{array}$ & $\begin{array}{l}\text { Binds to active site of thrombin (factor lla) and reversibly inhibits } \\
\text { free and clot-bound thrombin }\end{array}$ \\
\hline Prodrug & No & Yes \\
\hline Bioavailability & $>90 \%-95 \%$ & $6 \%-7 \%$ \\
\hline Protein binding & $99 \%$ & $35 \%$ \\
\hline Time to reach peak plasma levels & $72-96 \mathrm{hr}$ & $2-3 \mathrm{hr}$ \\
\hline Half-life & $36-44 \mathrm{hr}$ & $12-17 \mathrm{hr}$ \\
\hline Routine coagulation monitoring & Required, but frequency varies based on clinical situation & No requirement for routine monitoring \\
\hline Schedule & INR-adjusted, usually once daily & Fixed dose, once or twice daily \\
\hline Metabolism & CYP-450 hepatic microsomal enzymes, especially CYP2C9, CYP1A2, and CYP3A4 & Esterase-catalyzed hydrolysis in plasma or liver after intestinal P-gp transport \\
\hline Clearance & Almost entirely hepatic & $80 \%$ unchanged renally (after an intravenous dose), $20 \%$ hepatic after conjugation \\
\hline Drug interactions & $\begin{array}{l}\text { Drugs that affect CYP-450 hepatic microsomal enzymes and those } \\
\text { that displace warfarin from plasma proteins }\end{array}$ & P-gp inhibitors (CYP-450 system not involved) \\
\hline Antidote & Yes (vitamin K and plasma products) & No \\
\hline
\end{tabular}

Abbreviations: CYP-450, cytochrome P-450. INR, international normalized ratio; P-gp, P-glycoprotein. Modified from reference 9. 5,6,8,16,19,20-22,24,28,30,36,42-44

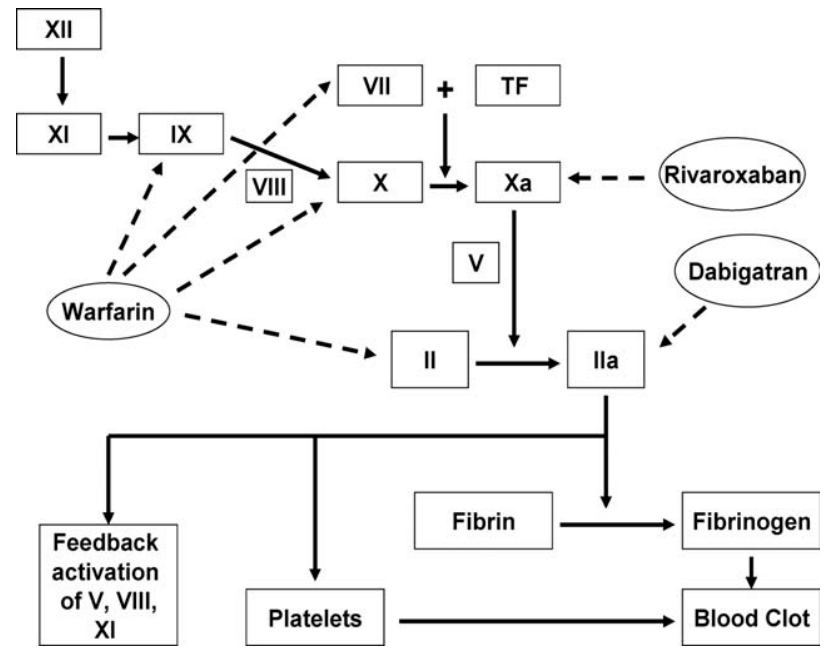

FIG. 1. Schema of intrinsic and extrinsic coagulation pathways showing the factor targets of warfarin, dabigatran, and rivaroxaban (a novel oral factor $\mathrm{Xa}$ inhibitor). Solid lines indicate activation; dashed lines indicate inhibition. Modified from references 2 and 24. Abbreviations: II, prothrombin; Ila, thrombin.

coagulation by converting fibrinogen to fibrin, amplifying its own generation by feedback activation of factors V, VIII, and XI, and by activating platelets (Figure 1). ${ }^{16}$ Dabigatran is a direct thrombin inhibitor that acts independently of anti-thrombin to inhibit both free and clot-bound thrombin. ${ }^{17,18}$ The bioavailability of dabigatran after oral intake is low $(6 \%-$ $7 \%) .{ }^{19-23}$ After absorption, the prodrug is rapidly converted by plasma and hepatic esterases to the active drug dabigatran, but it is not metabolized by the CYP-450 system, therefore reducing the potential for drug-drug interactions. ${ }^{8,23-28}$ The long half-life of dabigatran allows for once or twice daily dosing. ${ }^{21,24}$ The PK profile of dabigatran is predictable, with minimal inter- and intraindividual variation. ${ }^{21,22}$

Dabigatran is packaged in capsules that are hygroscopic. Therefore, the capsules should be stored in the original container with the cap tightly closed. Exposure of dabigatran capsules to air for prolonged peri- ods outside the original container can result in deterioration of the active compound and reduced efficacy. ${ }^{27,28}$ Dabigatran capsules contain tartaric acid which is necessary to facilitate dissolution of the medication in the gastrointestinal tract for optimal absorption. ${ }^{2}$ Breaking the capsules or removing the drug from the capsule can result in increased exposure. Therefore, dabigatran capsules should be taken intact, and patients should be instructed that dabigatran capsules should not be broken, chewed, or opened before administration. ${ }^{28}$ Alternative anticoagulants should be used if patients cannot swallow the capsule intact for any reason (eg, intubated patients).

\section{Dabigatran and Drug and Food Interactions}

Dabigatran acts as a substrate of the transporter protein P-glycoprotein (P-gp), which is also involved in the transport of many other drugs. ${ }^{5,16} \mathrm{P}$-gp is an efflux pump that functions to prevent the absorption of drugs in the intestine or increase the renal excretion of drugs that are P-gp substrates. ${ }^{25}$ Inhibitors of P-gp increase the serum concentrations of P-gp substrates, whereas P-gp inducers reduce the concentrations of these medications. ${ }^{13}$ Examples of P-gp inhibitors include clarithromycin, quinidine, and verapamil, whereas rifampin, pantoprazole, and St John's wort are known to induce P-gp. ${ }^{5,24,26}$ As an illustration, the coadministration of dabigatran and amiodarone, a known P-gp inhibitor, increases the area under the curve of drug plasma-concentration-time of dabigatran by $\approx 60 \%$ without significantly affecting levels of amiodarone. ${ }^{5,27}$ Nevertheless, dagibatran's prescribing information in the United States advises that the "P-gp inhibitors ketoconazole, verapamil, amiodarone, quinidine, and clarithromycin do not require dose adjustments, although these results should not be extrapolated to other P-gp inhibitors." ${ }^{28}$ In addition, the manufacturer recommends generally avoiding the concomitant use of the potent P-gp inducer rifampin with dabigatran, whereas the European Medicines 
Agency advises caution in the coadministration of rifampin or St John's wort with dabigatran. ${ }^{27,28}$

Not all P-gp substrates result in clinically significant interactions with dabigatran (eg, digoxin, diclofenac, and atorvastatin). ${ }^{19,29}$ The use of nonsteroidal antiinflammatory drugs and aspirin may increase the risk of bleeding in patients using dabigatran. ${ }^{5,26,27}$ It is not recommended to coadminister certain anti-platelet agents (such as clopidogrel, prasugrel, or ticlopidine) with dabigatran. ${ }^{26,30}$ Although the use of proton pump inhibitors such as pantoprazole leads to a $\approx 30 \%$ decrease in the area under the curve of dabigatran, coadministration of pantoprazole and other proton pump inhibitors with dabigatran in clinical trials did not affect bleeding risk or efficacy. ${ }^{27}$ Attention to potential drug interactions with dabigatran is important, because dabigatran is not usually monitored. Food interactions with dabigatran appear to be low, and therefore dabigatran can probably be taken with or without food, but caution is advised given the limited postmarketing experience with dabigatran. ${ }^{30}$ An excellent review of drug and dietary interactions of dabigatran has been published recently. ${ }^{5}$

\section{Use of Dabigatran in Patients With Liver or Renal Impairment}

Approximately $80 \%$ of dabigatran is excreted, largely unchanged, by the kidneys in healthy subjects. ${ }^{19}$ Patients with severe renal impairment (creatinine clearance $[\mathrm{CrCL}], \leq 30 \mathrm{~mL} / \mathrm{min}$ ) were excluded from phase 3 trials that evaluated dabigatran. ${ }^{31-35}$ A small study in patients with renal impairment showed a linear correlation between renal function and renal clearance of dabigatran, with proportional increases in the anticoagulant effects of dabigatran with decreasing renal function. ${ }^{36}$ For patients on hemodialysis, $62 \%-$ $68 \%$ of the dose was removed. ${ }^{36}$ The authors recommended avoidance of dabigatran in severe renal impairment, and a dose reduction was recommended for moderate renal impairment (CrCL, 31-50 mL/ min). ${ }^{13,36}$ Despite exclusion of patients with CrCL of $\leq 30 \mathrm{~mL} / \mathrm{min}$ from all phase 3 trials of dabigatran and the relative contraindication of the use of dabigatran in this patient population, the US Food and Drug Administration (FDA) approved a reduced dose of 75 mg twice daily for patients with CrCL of $15-30 \mathrm{~mL} /$ min, but no dosing recommendations were made for patients with CrCL of $\leq 15 \mathrm{~mL} / \mathrm{min}$ or for patients on dialysis. ${ }^{13,28,36}$ We believe that dabigatran should be used with great caution in patients with $\mathrm{CrCl} \mathrm{15-30}$ $\mathrm{mL} / \mathrm{min}$ given the limited outcome data in these patients, and alternative anticoagulants should be strongly considered for these patients until more data are available.

Less than $20 \%$ of the dabigatran dose is conjugated in the liver and subsequently secreted in the biliary system. ${ }^{19,23}$ Stangier et al. showed that moderate hepatic impairment does not affect the PK/PD or safety profile of dabigatran and concluded that dabigatran can be given to those patients without dose adjustment. ${ }^{37}$ On the other hand, severe hepatic impairment (Child-Pugh class $\mathrm{B}$ or $\mathrm{C}$ cirrhosis) and an alanine aminotransferase level more than 2 to 3 times the upper limit of normal were used as exclusion criteria in most of the phase 3 trials that evaluated dabigatran. ${ }^{16,24,34,35,38}$ The hepatic toxicity noted with the first generation oral direct thrombin inhibitor, ximelagatran, has not been seen with dabigatran in clinical trials, although long-term postmarketing data are lacking. ${ }^{32,34,35,38-40}$

\section{The Effect of Dabigatran on Common Coagulation Laboratory Tests and Recommendations for Monitoring Dabigatran's Anticoagulant Effects}

Despite the predictable PK profile of dabigatran, its effects on common coagulation assays remain incompletely defined. ${ }^{41}$ Most patients on dabigatran will have a prolonged activated partial thromboplastin time (aPTT) even at trough concentrations, but not in a linear predictable fashion. ${ }^{19,20,21,36,41}$ Dabigatran has few and unpredictable effects on prothrombin time (PT) and INR, and therapeutic concentrations of dabigatran usually result in only modest elevations of PT/INR. ${ }^{21,42}$ Although thrombin time (TT) displays a good linear correlation with plasma concentrations of dabigatran, the reagents used to perform TT in most clinical laboratories are not standardized. Therefore, TT is better suited to detecting the presence of dabigatran rather than monitoring its anticoagulant effects. $^{24,42}$ Therefore, even a slightly prolonged aPTT or TT could reflect significant plasma dabigatran levels. The best assays for monitoring dabigatran are the ecarin clotting time (ECT), modified thrombelastographic evaluations of whole blood clot formation, and the Hemoclot ${ }^{\circledR}$ Thrombin Inhibitor assay, but these tests are limited by lack of standardization and limited clinical availablity. ${ }^{24,42,43}$

\section{EFFICACY OF DABIGATRAN}

In this section, we provide a brief review of the major phase 3 trials that evaluated dabigatran for different indications (see references 13, 16, and 24 for recent detailed reviews of the clinical trials of dabigatran).

\section{Dabigatran for Thromboprophylaxis in Patients with Atrial Fibrillation}

The Randomized Evaluation of Long-Term Anticoagulation Therapy (RE-LY) trial was a prospective, noninferiority, phase 3 study of dabigatran that was the basis for its FDA approval in patients with nonvalvular AF. ${ }^{35,44}$ In RE-LY, 18,113 AF patients with another thromboembolic risk factor were randomized to receive fixed doses of dabigatran $(110 \mathrm{mg}$ or 150 mg twice daily) or adjusted-dose warfarin. ${ }^{35}$ The median duration of follow-up was 2 years and the primary outcome was stroke or systemic embolism. The 
primary outcome occurred in $1.69 \%$ per year in the warfarin group versus $1.53 \%$ per year in the group receiving $110 \mathrm{mg}$ of dabigatran twice daily (relative risk with dabigatran, $0.91 ; 95 \%$ confidence interval $[\mathrm{CI}], 0.74-1.11 ; P<0.001$ for noninferiority) and $1.11 \%$ per year in the group receiving $150 \mathrm{mg}$ of dabigatran twice daily (relative risk, 0.66; 95\% CI, $0.53-0.82 ; P<0.001$ for superiority). The rate of major bleeding was $3.36 \%$ per year in the warfarin group versus $2.71 \%$ per year in the dabigatran 110 mg group $(P=0.003)$ and $3.11 \%$ per year in the dabigatran $150 \mathrm{mg}$ group $(P=0.31)$. Intracranial bleeds were significantly less common in both dabigatran groups than with warfarin. Major gastrointestinal bleeding rate was significantly higher in the dabigatran group at the 150-mg dose than in the warfarin group. The mortality rate was $4.13 \%$ per year in the warfarin group versus $3.75 \%$ per year with $110 \mathrm{mg}$ of dabiga$\operatorname{tran}(P=0.13)$ and $3.64 \%$ per year with $150 \mathrm{mg}$ of dabigatran $(P=0.051){ }^{35}$ The authors concluded that in patients with nonvalvular AF, dabigatran given at a dose of $110 \mathrm{mg}$ twice daily was not inferior to warfarin, and was associated with lower rates of major hemorrhage than warfarin. ${ }^{35}$ Dabigatran given at a dose of $150 \mathrm{mg}$ twice daily was associated with lower rates of stroke and systemic embolism than warfarin but had similar rates of major hemorrhage. ${ }^{35}$ These effects were maintained in patients with previous stroke or transient ischemic attack, and in these patients starting dabigatran with and without prior VKA treatment. ${ }^{45,46}$

\section{Dabigatran for Prevention of Venous Thromboembolism After Major Orthopedic Procedures}

Without thromboprophylaxis, the incidence of venous thromboembolism (VTE) following major orthopedic surgery is $40 \%-60 \% .{ }^{47}$ Nevertheless, many patients do not receive appropriate thromboprophylaxis after orthopedic surgery, in part due to the limitations of VKAs and the inconvenience of low molecular weight heparin (LMWH) injections. ${ }^{48}$

\section{RE-NOVATE Trial}

Oral dabigatran versus enoxaparin for thromboprophylaxis after primary total hip arthroplasty (RENOVATE trail) was a prospective, noninferiority phase 3 trial in which 3494 patients undergoing total hip replacement (THR) were randomized in doubleblind fashion to 28-35 days of dabigatran $220 \mathrm{mg}$ or $150 \mathrm{mg}$ once daily, starting with a half-dose 1-4 hours after surgery, or subcutaneous (SC) enoxaparin $40 \mathrm{mg}$ once daily, starting the evening before surgery. ${ }^{33}$ The primary efficacy outcome was the composite of total VTE (venographic or symptomatic) and death from all causes during treatment. The primary efficacy outcome occurred in $6.7 \%$ in the enoxaparin group versus $6.0 \%$ in the dabigatran $220 \mathrm{mg}$ group (absolute difference [AD], $-0.7 \%$; 95\% CI, $-2.9 \%$ to
$1.6 \%$ ) and $8.6 \%$ in the $150 \mathrm{mg}$ group (AD, $1.9 \%$; $95 \%$ CI, $-0.6 \%$ to $4.4 \%$ ). There was no significant difference in major bleeding with either dose of dabigatran compared with enoxaparin $(220 \mathrm{mg}, P=0.44$; $150 \mathrm{mg}, P=0.60)$. It was concluded that oral dabigatran was not inferior to enoxaparin for prevention of VTE after THR surgery, with a similar safety profile. $^{33}$

\section{RE-NOVATE II Trial}

Oral dabigatran versus enoxaparin for thromboprophylaxis after primary total hip arthroplasty (RENOVATE II trail) was a randomized, double-blind, noninferiority phase 3 trial that compared dabigatran versus SC enoxaparin for extended thromboprophylaxis in patients undergoing THR. ${ }^{38}$ A total of 2055 patients were randomized to 28-35 days of oral dabigatran, $220 \mathrm{mg}$ once daily, starting with a half-dose 1-4 hours after surgery, or SC enoxaparin $40 \mathrm{mg}$ once daily, starting the evening before surgery. The primary efficacy outcome was the same as that in the RE-NOVATE trial. The primary efficacy outcome occurred in $7.7 \%$ of the dabigatran group versus $8.8 \%$ of the enoxaparin group (risk difference, $-1.1 \% ; 95 \%$ CI,-3.8 to $1.6 \% ; P<0.0001$ for the prespecified noninferiority margin. Major VTE plus VTE-related death occurred in $2.2 \%$ of the dabigatran group versus $4.2 \%$ of the enoxaparin group (risk difference, $-1.9 \% ; 95 \% \mathrm{CI},-3.6 \%$ to $-0.2 \% ; P=$ $0.03)$. Major bleeding occurred in $1.4 \%$ of the dabigatran group and $0.9 \%$ of the enoxaparin group $(P=$ $0.40)$. It was concluded that extended prophylaxis with oral dabigatran $220 \mathrm{mg}$ once daily was not inferior to SC enoxaparin $40 \mathrm{mg}$ once daily for prevention of VTE after THR. The safety profiles were similar between the 2 arms. $^{38}$

\section{RE-MODEL Trial}

In the Oral dabigatran etexilate vs. subcutaneous enoxaparin for the prevention of venous thromblembloism after total knee replacement (RE-MODEL trail) phase 3 trial, 2076 patients who underwent total knee replacement (TKR) were randomized to receive dabigatran $150 \mathrm{mg}$ or $220 \mathrm{mg}$ once daily starting with a half-dose 1-4 hours after surgery, or SC enoxaparin $40 \mathrm{mg}$ once daily starting the evening before surgery, for 6-10 days. ${ }^{32}$ Patients were followed-up for 3 months. The primary efficacy outcome was a composite of total VTE (venographic or symptomatic) and mortality during treatment. The primary efficacy outcome occurred in $37.7 \%$ of the enoxaparin group versus $36.4 \%$ of the dabigatran $220 \mathrm{mg}$ group (AD, $-1.3 \% ; 95 \% \mathrm{CI},-7.3$ to 4.6$)$ and $40.5 \%$ of the 150 mg group ( $\mathrm{AD}, 2.8 \% ; 95 \% \mathrm{CI},-3.1$ to 8.7$)$. The incidence of major bleeding did not differ between the groups $(1.3 \%$ versus $1.5 \%$ and $1.3 \%$, respectively). The conclusion was that dabigatran (220 mg or 150 $\mathrm{mg}$ ) was not inferior to enoxaparin for prevention of 
VTE after TKR surgery and exhibited a similar safety profile. $^{32}$

\section{RE-MOBILZE Trial}

The oral thrombin inhibitor dabigatran etexilate vs the North American enoxaparin regimen for the prevention of venous thromboembolism after knee arthroplasty surgery (RE-MOBILIZE trail) was a phase 3 trial that randomized 1896 patients after unilateral TKR to receive dabigatran 220 or $150 \mathrm{mg}$ once daily versus enoxaparin $30 \mathrm{mg}$ SC twice daily after surgery. ${ }^{40}$ Dosing stopped at contrast venography, 12-15 days after surgery. Follow-up was for 3 months. The primary outcome was a composite of total VTE events and all-cause mortality during treatment. With respect to the primary outcome, dabigatran at 220 and $150 \mathrm{mg}$ showed inferior efficacy to enoxaparin, with VTE rates of $31 \%(P=0.02$ vs enoxaparin), $34 \%$ ( $P<0.001$ vs enoxaparin), and $25 \%$, respectively. Major bleeding was similar. It was concluded that dabigatran was inferior to the twicedaily North American enoxaparin regimen, probably because of the latter's more intense and prolonged dosing. ${ }^{40}$ It should be noted that the first dose of dabigatran in this study was given 6-12 hours after surgery, compared with 1-4 hours postoperatively in REMODEL, which may have contributed to the inferior outcome. ${ }^{32,40}$

\section{Dabigatran for Treatment of Acute VTE}

RE-COVER was a large, randomized, noninferiority phase 3 trial that randomized 2564 patients with acute symptomatic proximal lower extremity deep vein thrombosis or pulmonary embolism to 6 months of dabigatran $150 \mathrm{mg}$ twice daily or dose-adjusted warfarin (INR 2/3). ${ }^{34}$ All patients initially received parenteral anticoagulation (LMWH or unfractionated heparin $[\mathrm{UFH}]$ ) for a median of 9 days. Patients in the warfarin group spent $60 \%$ of the time in the therapeutic range. In the dabigatran arm, $2.4 \%$ had recurrent VTE versus $2.1 \%$ in the warfarin arm $(P<0.001$ for the prespecified noninferiority margin). Major bleeding occurred in $1.6 \%$ of patients in the dabigatran arm and $1.9 \%$ in the warfarin arm (hazards ratio, $0.82 ; 95 \%$ CI, $0.45-1.48)$. There was no difference in the other safety endpoints (acute coronary syndrome, abnormal liver function tests and deaths). Adverse events (especially gastrointestinal) leading to discontinuation of the study drug occurred in $9 \%$ of patients assigned to dabigatran and $6.8 \%$ of patients assigned to warfarin $(P=0.05)$. It was concluded that a fixed dose of dabigatran was not inferior to warfarin for treatment of VTE, with a similar safety profile. ${ }^{34}$ It is important to note that the first dose of dabigatran was given after a median of 9 days of parenteral anticoagulation therapy, so the findings of this study do not provide data regarding the use of dabigatran as initial monotherapy for acute VTE. ${ }^{34}$
The results of additional randomized trials evaluating the use of dabigatran for acute VTE treatment (RE-COVER II) and secondary prevention of VTE (RE-MEDY and RE-SONATE) are expected soon. ${ }^{16}$

\section{SAFETY OF DABIGATRAN}

Aside from the bleeding risks discussed earlier, the most commonly reported side effect of dabigatran was dyspepsia. Dyspepsia occurred twice as frequently in patients taking dabigatran versus warfarin in the RELY trial $(11.5 \%$ vs $5.8 \%) .{ }^{35}$ One possible explanation for the higher incidence of dyspepsia is the tartaric acid component in dabigatran capsules. ${ }^{2}$ In the RE-LY study, myocardial infarction occurred more commonly in the dabigatran arms $(0.72 \%$ with $110 \mathrm{mg}$ and $0.74 \%$ with $150 \mathrm{mg})$ than the warfarin arm $(0.53 \%$, $P=0.07$ and 0.048 , respectively). ${ }^{24,35}$ It has been postulated that this observation could be related to a greater efficacy of warfarin for the prevention of myocardial infarction rather than an adverse effect of dabigatran. ${ }^{2}$ There was no increase in acute coronary syndrome rates noted with dabigatran in the other phase 3 trials. ${ }^{32-34,38,40}$ No increased risk of elevated liver function test has been noted with dabigatran, but long-term data are unavailable. ${ }^{32,34,35,38}$

\section{MANAGEMENT OF SPECIAL SITUATIONS THAT MAY ARISE IN THE USE OF DABIGATRAN \\ Switching From Warfarin to Dabigatran and Vice Versa}

When converting patients from warfarin to dabigatran, it is recommended that dabigatran be started once the INR falls below the lower limit of the desired therapeutic range. Conversely, when switching from dabigatran to warfarin, the manufacturer recommends starting warfarin based on renal function (Table 2). It should be noted that because dabigatran can increase the INR, the INR will better reflect warfarin's effect after dabigatran has been stopped for at least 2 days. $^{27,28}$

\section{Bridging from Dabigatran to Parenteral Anticoagu- lants and Vice Versa}

For patients currently receiving a parenteral anticoagulant, the manufacturer recommends starting dabigatran $0-2$ hours before the next administration time for parenteral anticoagulants (eg, LMWH) or at the time of discontinuation for continuously infused parenteral drugs (eg, intravenous UFH). ${ }^{28}$ For patients currently taking dabigatran who are transitioning to a parenteral anticoagulant, it is recommended to wait 12 hours $(\mathrm{CrCl} \geq 30 \mathrm{~mL} / \mathrm{min})$ or 24 hours $(\mathrm{CrCl}<30$ $\mathrm{mL} / \mathrm{min}$ ) after the last dose of dabigatran before initiating treatment with a parenteral anticoagulant. ${ }^{27,28}$ 
TABLE 2. Suggested Guidelines for Switching from Dabigatran to Warfarin Based on Renal Function

\begin{tabular}{ll}
\hline $\mathrm{CrCL}(\mathrm{mL} / \mathrm{min})$ & Time of Warfarin Initiation \\
\hline$\geq 50$ & 3 d before discontinuing dabigatran \\
$31-50$ & 2 before discontinuing dabigatran \\
$15-30$ & $1 \mathrm{~d}$ before discontinuing dabigatran \\
$<15$ & No recommendations made
\end{tabular}

Abbreviation: $\mathrm{CrCL}$, creatinine clearance. Adapted from reference 28.

\section{Management of Dabigatran Before Elective and Urgent Invasive Procedures}

Patients who undergo invasive procedures in the presence of therapeutic levels of dabigatran are at an increased risk of bleeding. The manufacturer recommends holding dabigatran for at least 24 hours before elective surgery depending on the degree of renal impairment and the risk of bleeding. ${ }^{28}$ Table 3 lists recommendations on the timing of discontinuation of dabigatran before a procedure. If emergent/urgent surgery is necessary for a patient who is on dabigatran, the risk of bleeding should be weighed against the urgency of the intervention. ${ }^{28,42,44}$ As mentioned earlier, the ECT or the Hemoclot ${ }^{\circledR}$ Thrombin Inhibitor assay are the preferred tests for measurement of dabigatran effects, but they are not standardized or widely clinically available. Instead, prolongation of the TT (preferably) or the aPTT can be used to determine the presence of dabigatran. ${ }^{28,42}$

\section{Overdose and Toxicity With Dabigatran}

Accidental or intentional overdose, or accumulation of dabigatran due to renal impairment, may lead to hemorrhagic complications. Unlike warfarin and heparin, there is no antidote for dabigatran. There are no widely available, reliable laboratory tests to measure the anticoagulant activity of dabigatran, and evidence-based guidelines to manage dabigatran toxicity do not exist. Therefore, in the event of dabigatran toxicity, treatment is largely supportive. Management of toxicity is dependent on whether the overdose/ accumulation is accompanied by bleeding or not. For overdose, interventions include adequate diuresis and the use of activated charcoal to reduce the absorption of dabigatran (within 2 hours of ingestion). ${ }^{42}$ In the event of bleeding, proposed measures include application of mechanical pressure to the sites of bleeding and infusion of pro-coagulant blood products such as activated prothrombin complex concentrates (eg, FEIBA $\mathrm{VH}^{\circledR}$, Baxter) or recombinant human activated factor VIIa NovoSeven $^{\circledR}$, NovoNordisk) (reviewed in references 26 and 42). In lifethreatening situations, hemodialysis could be considered, because it can remove $\approx 60 \%$ of the drug within 2-3 hours. ${ }^{42}$ Hemoperfusion over a charcoal filter or large volume hemofiltration have also been suggested in extreme situations. ${ }^{27,28,36,42}$ Acknowl-
TABLE 3. Recommendations for Discontinuation of Dabigatran Before Elective Surgery According to Renal Function and Risk of Bleeding

\begin{tabular}{llll}
\hline & & \multicolumn{2}{l}{$\begin{array}{l}\text { Suggested Timing of Discontinuation } \\
\text { of Dabigatran Before Surgery }\end{array}$} \\
\cline { 3 - 4 } CrCL (mL/min) & Half-Life (hr) & $\begin{array}{l}\text { Standard Risk } \\
\text { of Bleeding }\end{array}$ & $\begin{array}{l}\text { High Risk of } \\
\text { Bleeding }\end{array}$ \\
\hline$>80$ & $13(11-22)$ & $24 \mathrm{hr}$ & $2-4 \mathrm{~d}$ \\
$51-80$ & $15(12-34)$ & $24 \mathrm{hr}$ & $2-4 \mathrm{~d}$ \\
$31-50$ & $18(13-23)$ & $48 \mathrm{hr}$ & $4 \mathrm{~d}$ \\
$\leq 30^{\dagger}$ & $27(22-35)$ & $2-5 \mathrm{~d}$ & $>5 \mathrm{~d}$
\end{tabular}

Abbreviation: CrCL, creatinine clearance. *Examples of surgeries associated with a high risk of bleeding include but are not limited to cardiac, neurosurgical, and abdominal procedures. Other procedures such as lumbar punctures may also require complete hemostatic function. Other factors such as age, comorbid conditions, and concomitant use of anti-platelet agent therapy modify the risk of bleeding. ${ }^{\dagger}$ Dabigatran is not recommended for these patients. Adapted from reference 42.

edging their limitations, the ECT, TT, or aPTT may be used to direct therapy. ${ }^{27,42}$

\section{Pregnancy and Dabigatran Therapy}

Dabigatran is a class C drug during pregnancy, and there are no studies of dabigatran in pregnant women. Animal studies with dabigatran showed decreased fertility of pregnant rats; therefore, the risks and benefits of dabigatran therapy during pregnancy should be weighed carefully. ${ }^{27,28,44}$

\section{CONCLUSIONS}

Dabigatran is a novel, oral direct thrombin inhibitor that exhibits several advantages over warfarin. The predictable pharmacokinetic profile and minimal food and drug interactions of dabigatran allow for a fixeddosing regimen and obviate the need for routine laboratory monitoring. However, this apparent advantage is also a disadvantage. The lack of a reliable method to monitor dabigatran makes it more difficult to assess compliance, measure the impact of drug interactions, evaluate for toxicity, and determine bona fide therapeutic failure versus noncompliance in the event of breakthrough thromboembolism. ${ }^{28,42}$ Other limitations of dabigatran include the lack of an antidote and the dependence on normal renal function for elimination, with the potential for drug accumulation and toxicity with renal impairment. The noninferiority design of the clinical trials that evaluated dabigatran, the absence of long-term safety and efficacy data, and issues related to the cost effectiveness of dabigatran should be considered when prescribing this agent. More studies are needed to assess dabigatran in special patient populations (eg, the elderly, patients with renal and hepatic impairment, pediatric and pregnant patients) and to better understand dabigatran-drug interactions.

As more novel oral anticoagulant agents, such as factor Xa inhibitors, become available for clinical use, comparative studies will need to be performed to better define the role of each agent for specific 
indications. In the future, it might be possible to tailor the choice of the oral anticoagulant to the individual patient not only on the basis of the clinical indication but also the specific patient characteristics and possible drug interactions. For example, rivaroxaban $\left(X\right.$ arelto $\left.{ }^{\circledR}\right)$ is an oral direct factor Xa that was recently approved in the United States for VTE thromboprophylaxis following orthopedic surgery and in patients with non-valvular atrial fibrillation. ${ }^{2}$ Similar to dabigatran, rivaroxaban exhibits predictable PK and PD that allow fixed once or twice daily dosing and obviate the need for routine monitoring of its anticoagulant effects. ${ }^{2,16}$ Unlike dabigatran, rivaroxaban is an active drug and not a prodrug, and has a significantly higher bioavailability than dabigatran $(>80 \%$ vs $6 \%) .{ }^{16}$ In addition, the levels of rivaroxaban can be affected by drugs that interfere with both P-gp and the hepatic CYP-450 system, compared with dabigatran, which is affected only by drugs that affect P-gp. 8,16

Disclosures: Michael B. Streiff is a consultant for sanofi-aventis, DaiichiSankyo, Eisai, and Jannsen Pharmaceutical, Inc.; has received research grants from Bristol-Myers Squibb and sanofi-aventis; has received lecture honoraria from sanofi-aventis and Ortho-McNeil; and has received payment to develop presentations from Ortho-McNeil.

\section{References}

1. Arepally G, Bauer KA, Bhatt DL, et al. The use of antithrombotic therapies in the prevention and treatment of arterial and venous thrombosis: a survey of current knowledge and practice supporting the need for clinical education. Crit Pathw Cardiol. 2010;9:41-48.

2. Ansell J. Warfarin versus new agents: interpreting the data. Hematology Am Soc Hematol Educ Program. 2010;2010:221-228.

3. Bungard TJ, Ghali WA, Teo KK, McAlister FA, Tsuyuki RT. Why do patients with atrial fibrillation not receive warfarin? Arch Intern Med. 2000;160:41-46.

4. Go AS, Hylek EM, Borowsky LH, Phillips KA, Selby JV, Singer DE. Warfarin use among ambulatory patients with nonvalvular atrial fibrillation: The anticoagulation and risk factors in atrial fibrillation (ATRIA) study. Ann Intern Med. 1999;131:927-934.

5. Walenga JM, Adiguzel C. Drug and dietary interactions of the new and emerging oral anticoagulants. Int J Clin Pract. 2010;64:956-967.

6. Visser LE, van Schaik RH, van Vliet M, et al. The risk of bleeding complications in patients with cytochrome P450 CYP2C9*2 or CYP2C9"3 alleles on acenocoumarol or phenprocoumon. Thromb Haemost. 2004;92:61-66.

7. Jeske WP, Walenga JM, Hoppensteadt DA, et al. Differentiating lowmolecular-weight heparins based on chemical, biological, and pharmacologic properties: implications for the development of generic versions of low-molecular-weight heparins. Semin Thromb Hemost. 2008;34:74-85.

8. Denas G, Pengo V. Emerging anticoagulants. Expert Opin Emerg Drugs. 2011;16:31-44.

9. Holbrook AM, Pereira JA, Labiris R, et al. Systematic overview of warfarin and its drug and food interactions. Arch Intern Med. 2005; 165:1095-1106.

10. Jones M, McEwan P, Morgan CL, Peters JR, Goodfellow J, Currie CJ. Evaluation of the pattern of treatment, level of anticoagulation control, and outcome of treatment with warfarin in patients with non-valvar atrial fibrillation: a record linkage study in a large british population. Heart. 2005;91:472-477.

11. White HD, Gruber M, Feyzi J, et al. Comparison of outcomes among patients randomized to warfarin therapy according to anticoagulant control: results from SPORTIF III and V. Arch Intern Med. 2007; 167:239-245.

12. Budnitz DS, Shehab N, Kegler SR, Richards CL. Medication use leading to emergency department visits for adverse drug events in older adults. Ann Intern Med. 2007;147:755-765.

13. Wittkowsky AK. New oral anticoagulants: a practical guide for clinicians. J Thromb Thrombolysis. 2010;29:182-191.

14. Apostolakis S, Lip GY, Lane DA, Shantsila E. The quest for new anticoagulants: from clinical development to clinical practice [published ahead of print June 14, 2010]. Cardiovasc Ther. 2010. doi: 10.1111/ j.1755-5922.2010.00160.x.
15. Wienen W, Stassen JM, Priepke H, Ries UJ, Hauel N. In-vitro profile and ex-vivo anticoagulant activity of the direct thrombin inhibitor dabigatran and its orally active prodrug, dabigatran etexilate. Thromb Haemost. 2007;98:155-162.

16. Eriksson BI, Quinlan DJ, Eikelboom JW. Novel oral factor xa and thrombin inhibitors in the management of thromboembolism. Annu Rev Med. 2011;62:41-57.

17. Weitz JI, Hudoba M, Massel D, Maraganore J, Hirsh J. Clot-bound thrombin is protected from inhibition by heparin-antithrombin III but is susceptible to inactivation by antithrombin III-independent inhibitors. J Clin Invest. 1990;86:385-391.

18. Weitz JI, Leslie B, Hudoba M. Thrombin binds to soluble fibrin degradation products where it is protected from inhibition by heparinantithrombin but susceptible to inactivation by antithrombin-independent inhibitors. Circulation. 1998;97:544-552.

19. Stangier J. Clinical pharmacokinetics and pharmacodynamics of the oral direct thrombin inhibitor dabigatran etexilate. Clin Pharmacokinet. 2008;47:285-295.

20. Stangier J, Clemens A. Pharmacology, pharmacokinetics, and pharmacodynamics of dabigatran etexilate, an oral direct thrombin inhibitor. Clin Appl Thromb Hemost. 2009;15(suppl 1):9S-16S.

21. Stangier J, Rathgen K, Stahle H, Gansser D, Roth W. The pharmacokinetics, pharmacodynamics and tolerability of dabigatran etexilate, a new oral direct thrombin inhibitor, in healthy male subjects. $\mathrm{Br} \mathrm{J}$ Clin Pharmacol. 2007;64:292-303.

22. Stangier J, Stahle H, Rathgen K, Fuhr R. Pharmacokinetics and pharmacodynamics of the direct oral thrombin inhibitor dabigatran in healthy elderly subjects. Clin Pharmacokinet. 2008;47:47-59.

23. Blech S, Ebner T, Ludwig-Schwellinger E, Stangier J, Roth W. The metabolism and disposition of the oral direct thrombin inhibitor, dabigatran, in humans. Drug Metab Dispos. 2008;36:386-399.

24. Ma TK, Yan BP, Lam YY. Dabigatran etexilate versus warfarin as the oral anticoagulant of choice? A review of clinical data. Pharmacol Ther. 2011;129:185-194.

25. DuBuske LM. The role of P-glycoprotein and organic anion-transporting polypeptides in drug interactions. Drug Saf. 2005;28:789-801.

26. Levy JH, Key NS, Azran MS. Novel oral anticoagulants: implications in the perioperative setting. Anesthesiology. 2010;113:726-745.

27. European Medicines Agency. Pradaxa (dabigatran etexilate) [product information]. Available at: http://www.ema.europa.eu/docs/en_GB/ document_library/EPAR_-_Product_Information/human/000829/WC 500041059.pdf. Accessed March 25, 2011.

28. Dabigatran medication guide. Available at: http://bidocs.boehringeringelheim.com/BIWebAccess/ViewServlet.ser?docBase = renetnt\&folderPath = /Prescribing \%20Information/PIs/Pradaxa/Pradaxa.pdf. Accessed March 15, 2011.

29. Stangier J, Rathgen K, Stahle H, Reseski K, Kornicke T, Roth W. Coadministration of dabigatran etexilate and atorvastatin: assessment of potential impact on pharmacokinetics and pharmacodynamics. Am I Cardiovasc Drugs. 2009;9:59-68.

30. Wrigley BJ, Lip GY, Shantsila E. Novel oral anticoagulants: the potential relegation of vitamin $\mathrm{K}$ antagonists in clinical practice. Int $\mathrm{J}$ Clin Pract. 2010;64:835-838.

31. Camm AJ. The RE-LY study: Randomized Evaluation of Long-term anticoagulant therapY: dabigatran vs. warfarin. Eur Heart J. 2009; 30:2554-2555.

32. Eriksson BI, Dahl OE, Rosencher N, et al. Oral dabigatran etexilate vs. subcutaneous enoxaparin for the prevention of venous thromboembolism after total knee replacement: the RE-MODEL randomized trial. J Thromb Haemost. 2007;5:2178-2185.

33. Eriksson BI, Dahl OE, Rosencher N, et al. Dabigatran etexilate versus enoxaparin for prevention of venous thromboembolism after total hip replacement: a randomised, double-blind, non-inferiority trial. Lancet. 2007;370:949-956.

34. Schulman S, Kearon C, Kakkar AK, et al. Dabigatran versus warfarin in the treatment of acute venous thromboembolism. N Engl J Med. 2009;361:2342-2352.

35. Connolly SJ, Ezekowitz MD, Yusuf S, et al. Dabigatran versus warfarin in patients with atrial fibrillation. N Engl J Med. 2009;361: 1139-1151.

36. Stangier J, Rathgen K, Stahle H, Mazur D. Influence of renal impairment on the pharmacokinetics and pharmacodynamics of oral dabigatran etexilate: an open-label, parallel-group, single-centre study. Clin Pharmacokinet. 2010;49:259-268.

37. Stangier J, Stahle H, Rathgen K, Roth W, Shakeri-Nejad K. Pharmacokinetics and pharmacodynamics of dabigatran etexilate, an oral direct thrombin inhibitor, are not affected by moderate hepatic impairment. J Clin Pharmacol. 2008;48:1411-1419.

38. Eriksson BI, Dahl OE, Huo MH, et al. Oral dabigatran versus enoxaparin for thromboprophylaxis after primary total hip arthroplasty (RE-NOVATE II). A randomised, double-blind, non-inferiority trial. Thromb Haemost. 2011;105:721-729.

39. Prandoni $P$, Taher A. Insights from the dabigatran versus warfarin trial in patients with venous thromboembolism (the RE-COVER trial). Expert Opin Pharmacother. 2010;11:1035-1037. 
40. RE-MOBILIZE Writing Committee, Ginsberg JS, Davidson BL, et al. Oral thrombin inhibitor dabigatran etexilate vs north american enoxaparin regimen for prevention of venous thromboembolism after knee arthroplasty surgery. J Arthroplasty. 2009;24:1-9.

41. Lindahl TL, Baghaei F, Blixter IF, et al. Effects of the oral, direct thrombin inhibitor dabigatran on five common coagulation assays. Thromb Haemost. 2011;105:371-378.

42. van Ryn J, Stangier J, Haertter S, et al. Dabigatran etexilate-a novel, reversible, oral direct thrombin inhibitor: interpretation of coagulation assays and reversal of anticoagulant activity. Thromb Haemost. 2010;103:1116-1127.

43. Sorensen B, Ingerslev J. A direct thrombin inhibitor studied by dynamic whole blood clot formation. haemostatic response to exvivo addition of recombinant factor VIIa or activated prothrombin complex concentrate. Thromb Haemost. 2006;96:446-453.

44. US Food and Drug Administration. Dabigatran drug approval history. Available at: http://www.accessdata.fda.gov/drugsatfda_
docs/nda/2010/022512Orig1s000TOC.cfm. Accessed March 20, 2011

45. Diener HC, Connolly SJ, Ezekowitz MD, et al. Dabigatran compared with warfarin in patients with atrial fibrillation and previous transient ischaemic attack or stroke: a subgroup analysis of the RE-LY trial. Lancet Neurol. 2010;9:1157-1163.

46. Ezekowitz MD, Wallentin L, Connolly SJ, et al. Dabigatran and warfarin in vitamin $\mathrm{K}$ antagonist-naive and -experienced cohorts with atrial fibrillation. Circulation. 2010;122:2246-2253.

47. Geerts WH, Bergqvist D, Pineo GF, et al. Prevention of venous thromboembolism: American College of Chest Physicians EvidenceBased Clinical Practice Guidelines (8th edition). Chest. 2008;133(6 suppl):381S-453S.

48. Eikelboom JW, Karthikeyan G, Fagel N, Hirsh J. American Association of Orthopedic Surgeons and American College of Chest Physicians guidelines for venous thromboembolism prevention in hip and knee arthroplasty differ: what are the implications for clinicians and patients? Chest. 2009;135:513-520. 